\title{
Trombose de artéria carótida interna relacionada a trauma de palato em criança
}

Internal carotid artery thrombosis related to trauma of palate in a child

\author{
Fernando de Souza Almeida', Márcia Harumi Hossotani ${ }^{1}$, Juliana Del Grossi Moura ${ }^{2}$
}

RESUMO

Objetivo: Relatar um caso de trombose de artéria carótida interna secundária relacionada a trauma de palato mole em criança.

Descrição do caso: Criança admitida com quadro de alteração do nível de consciência, sonolência, afasia e hemiplegia direita; tinha antecedente de trauma corto contuso leve em palato mole há oito horas. A investigação tomográfica evidenciou acidente vascular isquêmico secundário à interrupção do fluxo sanguíneo em território de artéria cerebral média esquerda. A arteriografia mostrou oclusão da artéria carótida interna imediatamente distal à sua origem, com aspecto radiológico de "ponta de lápis", obstruindo o fluxo sanguíneo na região. A paciente foi submetida à investigação para doença pró-trombótica e cardíaca, contudo, não foi detectada nenhuma alteração. A administração de enoxaparina em dose terapêutica por três semanas conduziu à melhora clínica progressiva. Após três semanas de seguimento, a paciente não mostrava sequelas motoras.

Comentários: As lesões intraorais são frequentes em crianças e a maioria evolui sem complicações. A trombose da artéria carótida interna é uma complicação rara, mas bem documentada destas lesões e decorre da compressão do vaso com trombogênese localizada. A taxa de mortalidade relatada é de $30 \%$ e as sequelas ocorrem em $85 \%$ dos casos.

Palavras-chave: acidente cerebral vascular; isquemia encefálica; trombose das artérias carótidas; traumatismo de palato; criança.

\section{ABSTRACT}

Objective: To describe a child with internal carotid artery thrombosis secondary to trauma of the soft palate.

Case description: Child presented with altered level of consciousness, drowsiness, aphasia, right hemiplegia, and a history of trauma, with mild concussion of the soft palate eight hours prior to admission. CT scan showed ischemic stroke secondary to interruption of blood flow in the area of the left middle cerebral artery. Arteriography showed occlusion of the carotid artery just distal to its origin, with radiological appearance of "pencil tip", obstructing blood flow in the region. Patient underwent investigation for pro-thrombotic and heart diseases, which was negative. The child received enoxaparin at therapeutic dose for three weeks, showing progressive clinical improvement. After three weeks, the patient did not show motor problems.

Comments: Intraoral lesions are common in children, but most of them evolve without complications. However, the internal carotid artery thrombosis is a rare but well documented complication of these lesions and results from the vessel compression with localized thrombogenesis. The mortality rate is about $30 \%$, and sequels are seen in $85 \%$ of cases.

Key-words: stroke; brain ischemia; carotid artery thrombosis; soft palate trauma; child.
Instituição: Universidade de Mogi das Cruzes (UMC); Hospital das Clínicas Luzia de Pinho Melo, Mogi das Cruzes, SP, Brasil

${ }^{1}$ Acadêmico do curso de Medicina da UMC, Mogi das Cruzes, SP, Brasil ${ }^{2}$ Médica especialista em Pediatria e Terapia Intensiva pela Universidade de São Paulo; Professora Adjunta da disciplina de Pediatria da Faculdade de Medicina da UMC, Mogi das Cruzes, SP, Brasil
Endereço para correspondência:

Juliana Del Grossi Moura

Rua Moacyr Andreucci, 210 - Vila Oliveira

CEP 08790-520 - Mogi das Cruzes/SP

E-mail: judelgrossi@terra.com.br

Conflito de interesse: nada a declarar 


\section{Introdução}

Os acidentes vasculares cerebrais (AVC) apresentam-se como causa importante de morbidade em todas as faixas etárias. $\mathrm{Na}$ faixa etária pediátrica, cerca de metade dos casos ocorre em crianças com doenças de base, principalmente nas portadoras de cardiopatias $^{(1)}$. Nos Estados Unidos, estima-se que ocorram 2,7 casos de AVC por 100.000 crianças/ano, com maior incidência no $1^{\circ}$ ano de vida ${ }^{(2)}$. Segundo a US National for Health Statistics, a mortalidade por doenças cerebrovasculares é de 3,1 por 100.000, em crianças abaixo de um ano; 0,4 por 100.000 para crianças entre um e quatro anos e 0,2 por 100.000 para aquelas entre cinco e 14 anos $^{(3)}$, existindo predominância pelo sexo masculino, como identificado pelo International Pediatric Stroke Study (IPSS), que envolveu 30 centros em dez países ${ }^{(4)}$.

A anemia falciforme é uma das causas mais comuns de AVC na infância, sendo a variante homozigótica da hemoglobina S (HbSS), a mais recorrente para esse evento ${ }^{(5)}$. Embora seja incomum, com incidência em torno de $1 \%$, o AVC também pode ser o primeiro sinal da síndrome da imunodeficiência adquirida (SIDA) na infância ${ }^{(6)}$.

Os fatores de risco exibem variabilidade geográfica e étnica. Múltiplas variáveis podem coexistir e se somar, como traumas, infecções, predisposição genética, fatores pró-trombóticos, arteriopatias e doenças cardíacas, que permanecem como os principais fatores de risco para o AVC. Eventos pontuais podem desencadear fatores de risco preexistentes. Vários casos de AVC isquêmico estão associados diretamente a vasculopatias ou distúrbios hematológicos ou, ainda, podem se associar a distúrbios metabólicos e/ou outras síndromes genéticas ${ }^{(7)}$. Eventos genéticos raros estão relacionados ao AVC, como a displasia fibromuscular e síndrome de $\operatorname{Marfan}^{(8)}$, além das doenças de Menkes e de Fabry. O impacto da elevação epidêmica dos índices de obesidade na infância sobre a incidência de AVCé algo ainda a ser elucidado, porém os mesmos fatores de risco para o AVC na vida adulta, como dislipidemias, diabetes e hipertensão arterial podem exercer uma função significativa na faixa etária pediátrica ${ }^{(4)}$.

Quando relacionados à trombose de artéria carótida interna, os sinais e sintomas podem surgir de uma a 60 horas após o evento lesivo, sendo a artéria cerebral média a mais acometida, resultando em hemiplegia contralateral, hemianopsia homônima e afasia ${ }^{(9)}$. Já nos casos de AVC hemorrágico, os sintomas relacionados à hipertensão intracraniana são mais evidentes, sendo frequentes as alterações do nível de consciência, cefaléia, vômitos e coma ${ }^{(7)}$.

Nos pacientes com AVC após trauma oral, evento bastante raro, é imperativo que se descartem outras enfermidades e que se investiguem doenças congênitas ou adquiridas cardíacas, alterações vasculares, processos infecciosos vigentes, medicamentos em uso e, principalmente, distúrbios sanguíneos, que podem predispor a tal evento na presença de um fator desencadeante, como o trauma.

Neste contexto, o objetivo deste trabalho foi relatar um caso de trombose da artéria carótida interna relacionada com trauma de palato mole em criança, evento raro e pouco conhecido pela classe médica pediátrica.

\section{Descrição do caso}

O presente estudo foi aprovado pelos Comitês de Pesquisa e Ética do Hospital das Clínicas Luzia de Pinho Melo, e da Universidade de Mogi das Cruzes e teve o consentimento dos pais, de forma livre e esclarecida.

Criança de cinco anos de idade, sexo masculino, previamente hígida, apresentou-se no setor de emergência com história de ferimento em palato mole promovido por baqueta plástica (tambor infantil), após saltar de altura de cerca de um metro com o objeto dentro da cavidade oral; apresentava somente sangramento local de pequena intensidade. Na unidade de emergência, foi constatada a presença de ferimento corto-contuso em palato mole, com cerca de $2 \mathrm{~cm}$ e foi realizada a sutura da lesão. O paciente recebeu alta hospitalar com orientações de higiene e cuidados locais.

Cerca de oito horas depois, a criança retornou ao serviço de emergência com quadro de alteração do nível de consciência, sonolência, afasia e hemiplegia direita; foi encontrada em tal estado pela mãe, quando tentava acordá-la pela manhã. A responsável negava ocorrência de febre ou vômitos, relatando que notou enurese noturna, evento incomum para o paciente em questão.

O paciente foi admitido na Unidade de Terapia Intensiva com Glasgow de 9, hemiparético à direita, com contato verbal muito prejudicado; foi realizada tomografia de crânio que inicialmente não demonstrou alterações. Foi submetido ainda à coleta de líquor, com resultado normal. Nova tomografia feita após cerca de seis horas demonstrou imagens de hipodensidade frontal e de cápsula interna esquerda (Figura 1), sem efeito de massa, aventando-se a hipótese diagnóstica de acidente vascular encefálico em território de artéria cerebral média esquerda. Foi realizada angiografia digital cerebral, na qual foi observada oclusão da artéria carótida interna imediatamente distal à sua origem, com aspecto radiológico de "ponta de lápis" (Figura 2). O aspecto da imagem indicava a hipótese de dissecção arterial.

$\mathrm{Na}$ investigação complementar de doenças de base, foram realizados exames para triagem de comorbidades relacionadas 
à doença pró-trombótica ou cardíaca (fator VIII, antitrombina III; proteína S, anticorpo anticardiolipina, eletroforese de hemoglobina, ecocardiograma), além de exames gerais, como perfil hematimétrico, hepático, ionograma e função renal, nos quais não foi detectada nenhuma alteração.

Durante a internação, o paciente recebeu enoxaparina em dose terapêutica, evoluindo com melhora clínica progressiva,

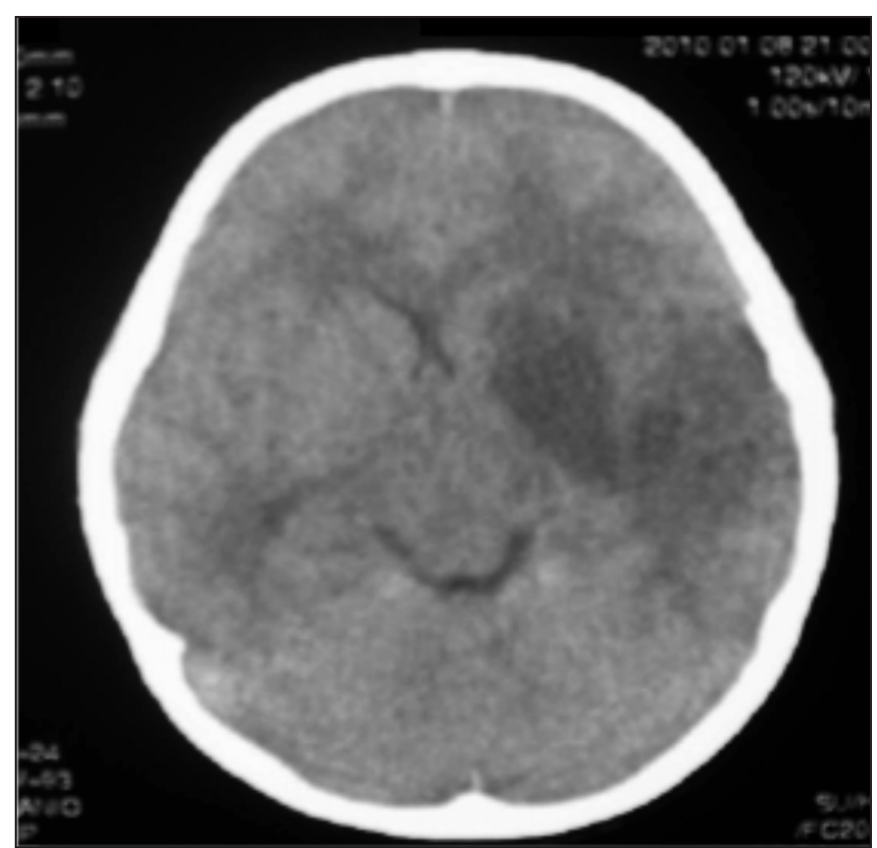

Figura 1 - Tomografia computadorizada de crânio. Imagem com sinais de hipodensidade frontal e de cápsula interna esquerda, realizada cerca de seis horas após a admissão hospitalar porém mantendo déficit motor e de fala importantes. Recebeu alta hospitalar e, durante seguimento ambulatorial, apresentou melhora significativa da hemiparesia direita e do quadro de afasia. Fez uso de anticoagulantes por três semanas. Após três semanas de seguimento, o paciente não apresentou sequelas motoras do evento em questão, mantendo dificuldade discreta em concatenar frases completas. A criança fazia uso de medicações.

\section{Discussão}

As lesões intraorais são comuns em crianças e as causas catalogadas incluem objetos pontiagudos como canetas, pirulitos, brinquedos, entre outros ${ }^{(10)}$. A maioria das lesões orais é mínima e não incorre em consequências mais graves. Estudos realizados por Radkowski ${ }^{(11)}$ e Hellman ${ }^{(12)}$, que revisaram casos de trauma oral, demonstraram que a maior parte dos pacientes evoluiu sem complicações significativas, além de sangramento de pequena intensidade e dor local. Disfagia, disfonia, sialorreia, ou enfisema subcutâneo podem sugerir laceração na região posterior da orofaringe ${ }^{(13)}$. Entretanto, o potencial para uma complicação neurológica devastadora existe em qualquer trauma penetrante de palato, mesmo mínimo. Os relatos apontam para uma taxa de mortalidade de $30 \%$, e sequelas permanentes em $85 \%$ das crianças acometidas ${ }^{(14,15)}$.

A trombose da artéria carótida interna com déficit neurológico subsequente é uma complicação bem documentada dos ferimentos corto-contusos desta região. Quando a mesma é lesada, desenvolvem-se irritabilidade, vômitos e sonolência.

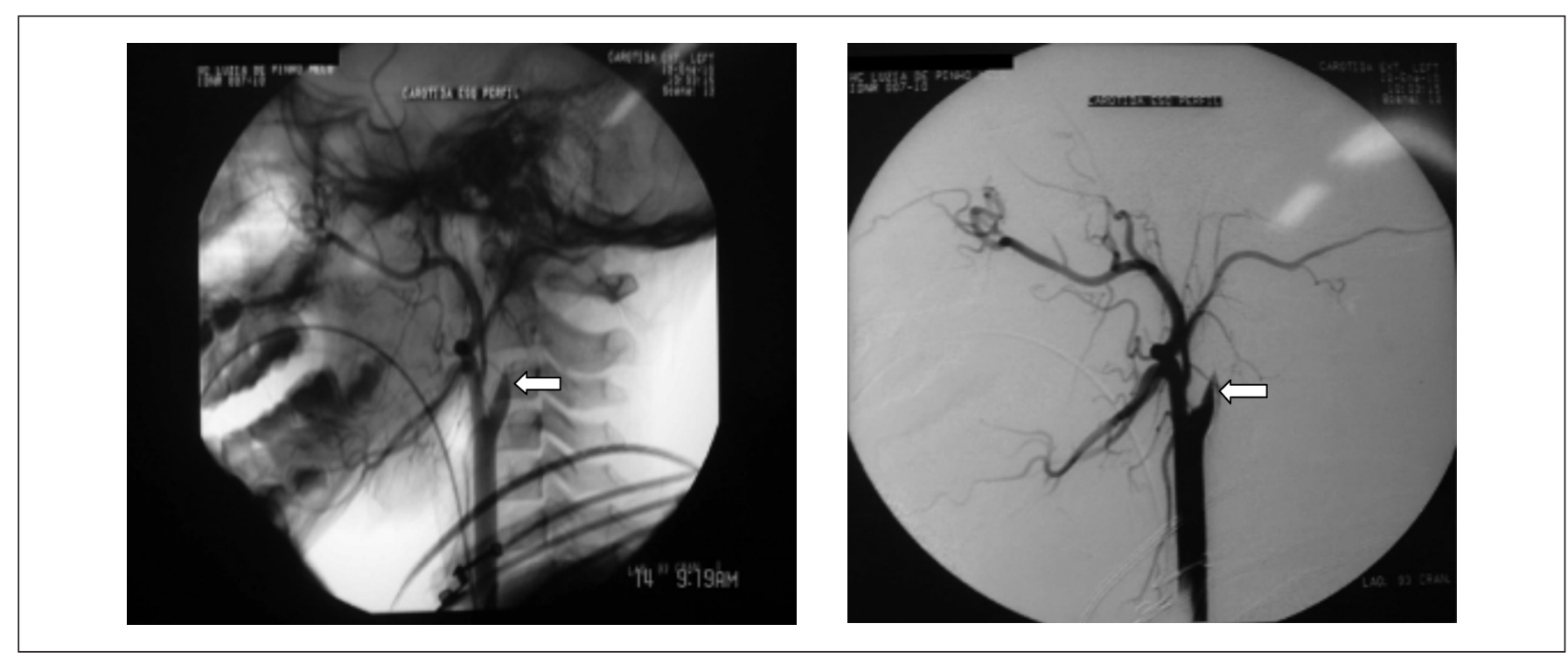

Figura 2 - Angiografia digital cerebral. Imagens demonstrando oclusão da artéria carótida interna esquerda, imediatamente distal a sua origem, com aspecto radiológico de ponta de lápis, identificado pelas setas 
Os sintomas neurológicos, em geral, se manifestam entre duas a 48 horas após a lesão ${ }^{(16,17)}$.

Frequentemente não há penetração do vaso em si, mas há compressão da artéria carótida interna contra a vértebra ou a base do crânio, causando uma lesão da camada íntima que ocasiona um processo localizado de trombogênese. Usualmente, existe um intervalo lúcido de quatro a 48 horas entre a lesão inicial e o desenvolvimento da lesão neurológica. Este atraso é causado pelo tempo para formação e propagação do trombo intraluminal ou a sua embolização.

Nos casos típicos, ocorre acometimento da artéria cerebral média, resultando em hemiparesia contralateral. Devido ao aspecto radiológico obtido, estas lesões também são denominadas "lesões em ponta de lápis" (pencil point lesions). Outros sinais incluem visão borrada, hemianopsia, afasia, hemiparesia ou hemiplegia, crises convulsivas motoras focais e depressão sensorial. Uma grande área de infarto, edema progressivo e efeito de massa com herniação transtentorial podem ocorrer quando o trombo se propaga pelas artérias cerebrais média e anterior. No entanto, a presença da trombose é evento de exceção e o período mínimo de observação de 48 horas pode não ser suficiente nos casos de lesão estabelecida, pois existem relatos de ocorrência de sintomas até 96 horas após o trauma ${ }^{(10)}$. Outro ponto importante é a viabilidade financeira deste período de observação, que acarretaria em custos hospitalares elevados,

\section{Referências bibliográficas}

1. Domi T, Edgell DS, McCrindle BW, Williams WG, Chan AK, MacGregor DL et al. Frequency, predictors, and neurologic outcomes of vaso-occlusive strokes associated with cardiac surgery in children. Pediatrics 2008;122:1292-8.

2. Chung B, Wong V. Pediatric stroke among Hong Kong Chinese subjects. Pediatrics 2004;114:e206-12.

3. Mallick AA, O'Callaghan FJ. The epidemiology of childhood stroke. Eur J Paediatr Neurol 2010;14:197-205.

4. Lo W, Stephens J, Fernandez S. Statement of correction: pediatric stroke in the United States and the impact of risk factors. J Child Neurol 2009;24:194-203.

5. Angulo IL. Stroke and other vascular complications of the Central Nervous System in sickle cell disease. Rev Bras Hematol Hemoter 2007;29:262-7.

6. Rocha C, Gouvêa AT, Machado DM, Hörnke L, Succi RC. Ischaemic stroke in two children with HIV-1. Arq Neuropsiquiatr 2003;61:1015-7.

7. Calder K, Kokorowski P, Tran T, Henderson S. Emergency department presentation of pediatric stroke. Pediatr Emerg Care 2003;19:320-8.

8. Moriarty JM, Lukas C, Rossler L, Thiels C, Drescher R. Carotid artery dissection following a minor household accident in a 10-month-old child. Ir J MedSci 2009; 178:535-9.

9. Borges G, Ramina R, Fernandes YB, Zambelli HJ, Marques EL, Menezes JR et al. Thrombosis of the internal artery secondary to soft palate injury: case report. Arq Neuropsiquiatr 1999;57:1027-31. considerando-se a alta incidência de trauma intraoral na faixa etária pediátrica.

Desta forma, a admissão hospitalar estaria reservada para as crianças que apresentarem sintomas neurológicos precoces, situação social de risco ou quando houver a necessidade de procedimento cirúrgico. Para os demais pacientes, deve-se orientar os sinais de alerta para retorno imediato ao serviço de emergência (irritabilidade, vômitos, sonolência, sinais focais, crises convulsivas).

O tratamento também é controverso; alguns estudos não encontraram diferença no prognóstico entre pacientes submetidos a tratamento com anticoagulantes e aqueles que não receberam tratamento específico ${ }^{(16,17)}$. A abordagem cirúrgica tem obtido resultado variável, com boas taxas de sucesso quando os déficits neurológicos iniciais são mínimos ${ }^{(18)}$.

Nesse caso, a trombose de artéria carótida interna ocorreu em uma criança de cinco anos de idade, após trauma banal de orofaringe, sem nenhum indício inicial de gravidade, cerca de oito horas após o evento traumático. Apesar de ser um evento raro, o AVC secundário ao trauma orofaríngeo em crianças é um diagnóstico bem estabelecido e exige do pediatra um conhecimento da causa base, além de um alto grau de suspeita. Outro ponto importante é a necessidade de conhecimento deste evento para orientação de familiares e responsáveis quanto aos sinais de alerta e necessidade de retorno ao departamento de emergência quando da sua presença.

10. Joseph MM, Lewis S. Stroke after penetrating trauma of the oropharynx Pediatr Emerg Care 2002;18:179-81.

11. Radkowski D, McGill TJ, Healy GB, Jones DT. Penetrating trauma of the oropharynx in children. Laryngoscope 1993;103:991-4.

12. Hellmann JR, Shott SR, Gootee MJ. Impalement injuries of the palate in children: review of 131 cases. Int $\mathrm{J}$ Pediatr Otorhinolaryngol 1993;26:157-63.

13. Singer JI. Management strategy for penetrating oropharyngeal injury. Pediatr Emerg Care 1989;5:250-2.

14. Graham CJ, Schwartz JE, Stacy T. Stroke following oral trauma in children. Ann Emerg Med 1991;20:1029-31.

15. Rowley H, Christian J, Dennis A. Pharyngeal perforation: an easily missed finding following intra-oral injury. J Accid Emerg Med 1995;12:145-6.

16. Mains B, Nagle M. Thrombosis of the internal carotid artery due to soft palate injury. J Laryngol Otol 1989;103:796-7.

17. Woodhurst WB, Robertson WD, Thompson GB. Carotid injury due to intraoral trauma: case report and review of the literature. Neurosurgery 1980;6:559-63.

18. Higgens GL 3rd, Meredith JT. Internal carotid artery thrombosis following penetrating trauma of the soft palate: an injury of youth. J Fam Pract 1991;32:316-22 